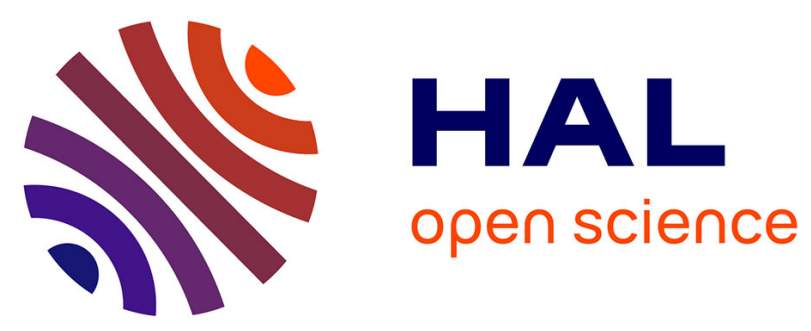

\title{
Fabrication of Bi2Te3 nanowire arrays and thermal conductivity measurement by $3 \omega$-scanning thermal microscopy
}

M. Muñoz Rojo, Stéphane Grauby, Jean-Michel Rampnoux, O. Caballero-Calero, M. Martin-Gonzalez, Stefan Dilhaire

\section{To cite this version:}

M. Muñoz Rojo, Stéphane Grauby, Jean-Michel Rampnoux, O. Caballero-Calero, M. Martin-Gonzalez, et al.. Fabrication of Bi2Te3 nanowire arrays and thermal conductivity measurement by $3 \omega$-scanning thermal microscopy. Journal of Applied Physics, 2013, 113 (5), pp.054308 (1-7). 10.1063/1.4790363 . hal-00805572

\section{HAL Id: hal-00805572 \\ https://hal.science/hal-00805572}

Submitted on 8 Mar 2018

HAL is a multi-disciplinary open access archive for the deposit and dissemination of scientific research documents, whether they are published or not. The documents may come from teaching and research institutions in France or abroad, or from public or private research centers.
L'archive ouverte pluridisciplinaire HAL, est destinée au dépôt et à la diffusion de documents scientifiques de niveau recherche, publiés ou non, émanant des établissements d'enseignement et de recherche français ou étrangers, des laboratoires publics ou privés. 


\section{AIP Applied Physics}

\section{Fabrication of $\mathrm{Bi} 2 \mathrm{Te} 3$ nanowire arrays and thermal conductivity measurement by $3 \omega$-scanning thermal microscopy}

M. Muñoz Rojo, S. Grauby, J.-M. Rampnoux, O. Caballero-Calero, M. Martin-Gonzalez et al.

Citation: J. Appl. Phys. 113, 054308 (2013); doi: 10.1063/1.4790363

View online: http://dx.doi.org/10.1063/1.4790363

View Table of Contents: http://jap.aip.org/resource/1/JAPIAU/v113/i5

Published by the American Institute of Physics.

\section{Related Articles}

Semiconducting selenium nanoparticles: Structural, electrical characterization, and formation of a back-to-back

Schottky diode device

J. Appl. Phys. 113, 123704 (2013)

Magnetoimpedance studies in as quenched Fe73.5Si13.5B8CuV3-xAINbx nanocrystalline ribbons J. Appl. Phys. 113, 17A321 (2013)

Green luminescence from Cu-doped $\mathrm{ZnO}$ nanorods: Role of $\mathrm{Zn}$ vacancies and negative thermal quenching Appl. Phys. Lett. 102, 111106 (2013)

Formation of graphene nano-particle by means of pulsed discharge to ethanol

J. Appl. Phys. 113, 114304 (2013)

Atomic structure of tensile-strained $\mathrm{GaAs} / \mathrm{GaSb}(001)$ nanostructures

Appl. Phys. Lett. 102, 102105 (2013)

\section{Additional information on J. Appl. Phys.}

Journal Homepage: http://jap.aip.org/

Journal Information: http://jap.aip.org/about/about_the_journal

Top downloads: http://jap.aip.org/features/most_downloaded

Information for Authors: http://jap.aip.org/authors

\section{ADVERTISEMENT}

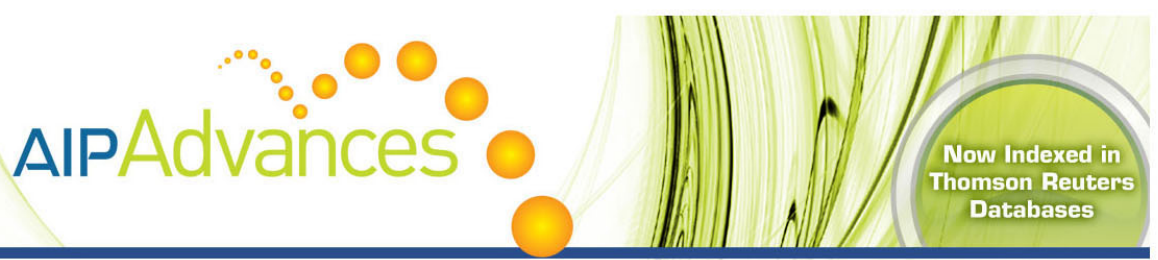

\section{Explore AIP's open access journal: - Rapid publication \\ - Article-level metrics \\ Post-publication rating and commenting}




\title{
Fabrication of $\mathrm{Bi}_{2} \mathrm{Te}_{3}$ nanowire arrays and thermal conductivity measurement by $3 \omega$-scanning thermal microscopy
}

\author{
M. Muñoz Rojo, ${ }^{1}$ S. Grauby, ${ }^{2, a)}$ J.-M. Rampnoux, ${ }^{2}$ O. Caballero-Calero, ${ }^{1}$ \\ M. Martin-Gonzalez, ${ }^{1}$ and S. Dilhaire ${ }^{2}$ \\ ${ }^{1}$ Instituto de Microelectrónica de Madrid, IMM-CSIC, 8 PTM 28760 Tres Cantos, Madrid, Spain \\ ${ }^{2}$ Univ. Bordeaux, LOMA, UMR 5798, 33405 Talence, France
}

(Received 23 November 2012; accepted 21 January 2013; published online 6 February 2013)

\begin{abstract}
$\mathrm{Bi}_{2} \mathrm{Te}_{3}$ is well-known for its utility in thermoelectrical applications and more recently as topological insulator. Its nanostructuration has attracted plenty of attention because of its potential capacity to reduce thermal conductivity. Here, we have grown a composite sample made of a $\mathrm{Bi}_{2} \mathrm{Te}_{3}$ nanowires (NWs) array embedded in an alumina matrix. We have then performed scanning thermal microscopy (SThM) in a $3 \omega$ configuration to measure its equivalent thermal resistance. Using an effective medium model, we could then estimate the mean composite thermal conductivity as well as the thermal conductivity of the NWs to be, respectively, $\left(\lambda_{\mathrm{C}}\right)=(1.68 \pm 0.20) \mathrm{W} / \mathrm{mK}$ and $\left(\lambda_{\mathrm{NW}}\right)=(1.37 \pm 0.20) \mathrm{W} / \mathrm{mK}$, showing a slight thermal conductivity reduction. Up to now, there have been two main techniques reported in literature to evaluate the thermal conductivity of nanostructures: the use of a thermal microchip to probe a single NW once its matrix has been dissolved or the probing of the whole NWs array embedded in a matrix, obtaining the thermal conductivity of the whole as an effective medium. However, the $3 \omega-\mathrm{SThM}$ presented here is the only technique able to measure the thermal conductivity of single NWs embedded in a matrix as well as the thermal conductivity of the composite locally. This technique is more versatile and straightforward than other methods to obtain the thermal conductivity of nanostructures. (C) 2013 American Institute of Physics. [http://dx.doi.org/10.1063/1.4790363]
\end{abstract}

\section{INTRODUCTION}

Over the past decade, one dimensional structures such as nanowires $(\mathrm{NWs})^{1-3}$ or nanotubes ${ }^{4,5}$ have experienced an important development because of their potential thermoelectric applications. Indeed, nanostructured materials for which at least one dimension is smaller than the phonon mean free path and larger than the electron mean free path are expected to offer a reduction of their thermal conductivity $\lambda$ (Refs. 6 and 7) without modifying much their power factor $\left(S^{2} \sigma\right)$ where $S$ and $\sigma$ are the Seebeck coefficient and electrical conductivity, respectively. The thermal conductivity reduction can be explained by the alteration of the phonon transport at the nanometric scale because there appear several effects, such as changes in the phonon dispersion relation and increased phonon boundary scattering. ${ }^{7-10}$ This kind of structures then behaves as a phonon glass and an electron crystal and is expected to exhibit a high figure of merit $Z T=\frac{S^{2} \sigma}{\lambda} T$ (with $\mathrm{T}$ the absolute temperature) which quantifies their thermoelectric efficiency. Then, onedimensional systems such as NWs can be very good candidates for thermoelectric applications.

There is hence a real double challenge: the first one is the development of a chemical process able to control the composition, size, spacing, and surface state of the NWs; the second one is the measurement of the thermal conductivity of those nanometric structures. As an example, several theo-

\footnotetext{
a) Author to whom correspondence should be addressed. Electronic mail: stephane.grauby@u-bordeaux1.fr.
}

retical studies ${ }^{7,11,12}$ have predicted that the thermal conductivity of silicon NWs could be largely reduced by their nanostructuration. Experiments ${ }^{13,14}$ have lead to an estimation of a thermal conductivity lower than $10 \mathrm{~W} / \mathrm{mK}$ for silicon NWs, leading to a ZT around 0.6 at room temperature. ${ }^{14}$ As bulk $\mathrm{Bi}_{2} \mathrm{Te}_{3}$ is the most commonly used thermoelectric material, its nanostructuration can let us hope promising results. ${ }^{15-17}$ Therefore, for this work, we have grown $\mathrm{Bi}_{2} \mathrm{Te}_{3}$ NWs by electrochemical deposition. These NWs are embedded in a porous alumina template and present different diameters.

The characterization of NW properties, such as structural, electrical, or thermal properties, is of vital importance. Focusing our attention in thermal characterization, we observe that there is a need of techniques able to reach a nanometric lateral spatial resolution. Optical methods, such as infrared thermometry, visible thermo-reflectance, or interferometry, which are diffraction limited, cannot reach this resolution. Since its invention in 1986, the scanning thermal microscopy (SThM) ${ }^{18-20}$ is presented as the most efficient technique to study thermal transport in nano-objects and nanomaterials. $^{21}$

In this work, we present measurements of the thermal conductivity carried out by $3 \omega$-SThM on a composite sample made of $\mathrm{a} \mathrm{Bi}_{2} \mathrm{Te}_{3} \mathrm{NWs}$ array embedded in an alumina matrix. To date, most of the NW thermal conductivity measurements have been taken for single NWs. ${ }^{13,14}$ However, many future NW devices will be based on a large number of NWs embedded in a matrix. Therefore, thermal properties of an array of NWs might be different to single ones because of 
the matrix-NWs interaction. These changes in the thermal conductivity depend on the NW diameter and the geometry of the array as well as the character of the NW/matrix interface and the presence of additional components. ${ }^{22}$

We first present, in Sec. II, the sample we have studied as well as the $3 \omega$-SThM measurement method used. Then, we show in Sec. III, the topographical and thermal images obtained for the $\mathrm{Bi}_{2} \mathrm{Te}_{3}$ NWs sample and we show how we can extract the thermal conductivity of the composite $\mathrm{Bi}_{2} \mathrm{Te}_{3}$ NWs/alumina matrix. Finally, in Sec. IV, we discuss the possibility to deduce from the previous result the thermal conductivity of the NWs.

\section{SAMPLE AND SET-UP}

\section{A. Sample growing process and description}

Nominal $200 \mathrm{~nm}$ diameter $\mathrm{Bi}_{2} \mathrm{Te}_{3} \mathrm{NW}$ arrays were prepared in Whatman $(\mathrm{C}$ anodic alumina oxide (AAO) filters. One surface of the AAO templates was deposited with $5 \mathrm{~nm}$ of $\mathrm{Cr}$ and $150 \mathrm{~nm}$ of gold by e-beam evaporation. Then, an electrical contact was made with the gold layer and the AAO template was used as a working electrode to perform an electrodeposition in a three electrode cell configuration following a similar procedure than the one described in Refs. 23-25.

The deposition potential was set to $-20 \mathrm{mV}$ versus $\mathrm{Ag} /$ $\mathrm{AgCl}$ and the deposition has been carried out for $12 \mathrm{~h}$ until most of the $\mathrm{Bi}_{2} \mathrm{Te}_{3}$ wires growing inside the AAO pores reached the alumina template surface. Afterwards, the sample was polished using $50 \mathrm{~nm}$ alumina nanoparticles. In order to eliminate the possible excess of alumina nanoparticles from the polishing, the sample was treated afterwards with $10 \%$ weight in volume concentration of $\mathrm{KOH}$.

$37 \mu \mathrm{m}$ long $\mathrm{Bi}_{2} \mathrm{Te}_{3}$ NWs with a composition (measured by Energy Dispersive X-Ray spectroscopy) of $\mathrm{Bi}_{2.02 \pm 0.04} \mathrm{Te}_{2.98 \pm 0.06}$, the c-axis oriented perpendicular to the surface of the alumina (measured by X-Ray Diffraction), and diameters ranging from 200 to $400 \mathrm{~nm}$ were finally obtained to be measured by SThM in adequate conditions. Figure 1 presents scanning electron microscopy (SEM) images of the sample. We can see that not every pore is filled with a NW (Figure 1(a)) because some of the NWs are not long enough to reach the surface of the matrix (Figure 1(b)). In our case, given that the measurements are made using the tip, we access and analyze only the NWs jutting out above the matrix.

\section{B. Experimental set-up for thermal conductivity measurements}

Thermal measurements have been investigated using a $3 \omega$ SThM method ${ }^{26-28}$ at room temperature to carry out thermal images while simultaneously obtaining contact mode topographical images.

The tip used is a V-shaped $\mathrm{Pd} / \mathrm{SiO}_{2}$ thermo-resistive probe $^{29,30}$ which offers a nanometric topographic lateral resolution, a typical $100 \mathrm{~nm}$ thermal lateral resolution and $3 \mathrm{kHz}$ thermal cut-off frequency, ${ }^{30}$ far better than the classical Wollaston thermoresistive probe. ${ }^{28,31}$ It is made of a thin palladium $(\mathrm{Pd})$ ribbon on a micrometric thick $\mathrm{SiO}_{2}$ silica layer. The thin Pd ribbon acts as the thermo-resistive ele-

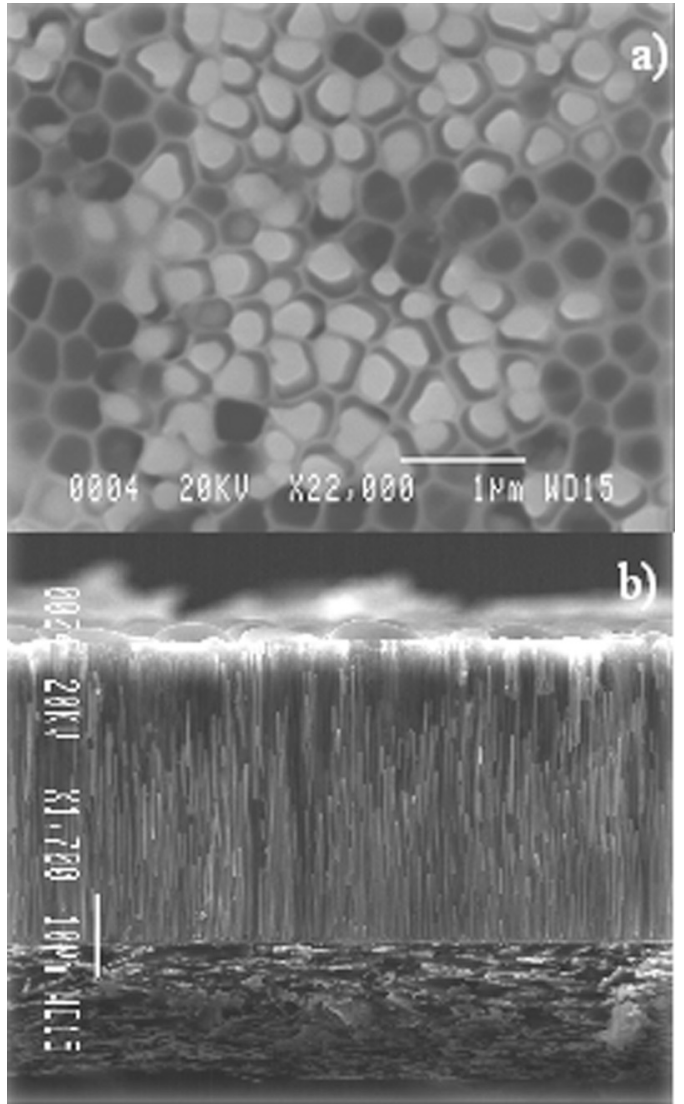

FIG. 1. Sample SEM pictures: (a) Top view of the Whatman alumina matrix partially filled with $\mathrm{Bi}_{2} \mathrm{Te}_{3}$ nanowires whose diameter ranges between $200 \mathrm{~nm}$ and $400 \mathrm{~nm}$, (b) Cross section view of the sample before polishing.

ment. Its temperature coefficient has been measured: $\alpha_{\text {tip }}=1.6 \times 10^{-3} \mathrm{~K}^{-1}$. The probe electrical resistance is $\mathrm{R}_{0}=327 \Omega$. The length and width of each arm of the V-shaped Pd tip are measured from SEM images to be typically $7 \mu \mathrm{m}$ and $3.5 \mu \mathrm{m}$, respectively.

For the $3 \omega$-SThM measurement, a sinusoidal current $\mathrm{I}(\mathrm{t})=\mathrm{I}_{0} \sin (\omega \mathrm{t})$, with $\omega$ being the fundamental pulsation, is passed through the thermoresistive probe. A heat flux $\mathrm{P}_{\text {Joule }}$ at pulsation $2 \omega$ is, therefore, dissipated in the probe, generating a temperature variation $\mathrm{T}_{2 \omega}$ at the same pulsation. The thermoresistive probe resistance is then modulated at $2 \omega$. Finally, according to Ohm's law, the tip voltage is modulated at $3 \omega$ and its amplitude can be expressed as ${ }^{12}$

$$
\left(V_{3 \omega}\right)_{T i p}=K_{a m p l i} \frac{R_{T i p} \alpha_{T i p} I_{0}}{2}\left\langle T_{2 \omega}\right\rangle,
$$

where $\mathrm{K}_{\text {ampli }}$ is the amplification system gain, $\mathrm{I}_{0}$ is the amplitude of the $\omega$ pulsation current supplying the probe, and $\left\langle T_{2 \omega}\right\rangle$ is the mean temperature variation amplitude over the tip length.

When the tip comes into contact with a material, a heat flow goes from the tip to the sample and this flow depends on the thermal conductance of the sample. Consequently, the $\left\langle T_{2 \omega}\right\rangle$ tip temperature variations depend on the equivalent thermal resistance $\mathrm{R}_{\mathrm{eq}}$ between the tip and the sample. The more conductive the sample, the lower the $2 \omega$ thermal variations. This configuration is hence sometimes called 


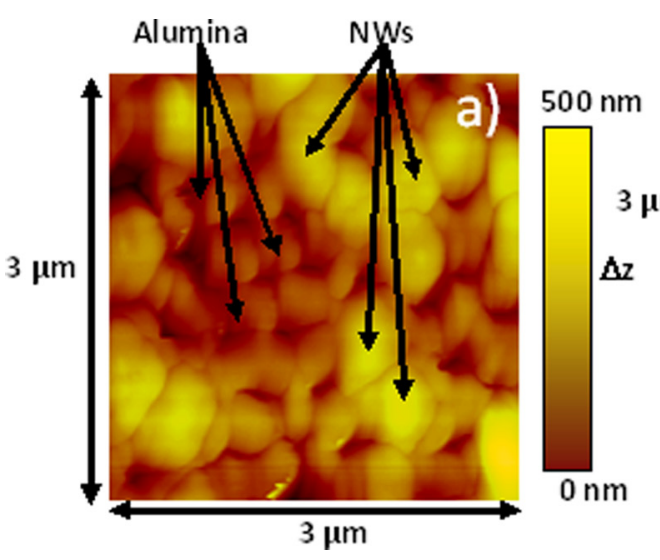

conductivity contrast imaging. Experimentally, the tip scans the sample and we measure the $3 \omega$ tip voltage $\left(V_{3 \omega}\right)_{\text {Tip }}$ using a lock-in amplifier. From the $\left(\mathrm{V}_{3 \omega}\right)_{\text {Tip }}$ image, we can then deduce a $\left\langle T_{2 \omega}\right\rangle$ tip temperature variations map and then an equivalent tip-sample thermal resistance $R_{\text {eq }}$ image. ${ }^{29}$

Let us underline that, for a sample constituted of several NWs or a NW "carpet," this experimental method enables to simultaneously measure $\mathrm{R}_{\mathrm{eq}}$ on each $\mathrm{NW}$ of the thermal image. Then we can do a statistical data processing and thus deduce a mean thermal conductivity and its associated standard deviation.

As a preliminary and necessary measurement, we have experimentally estimated the tip-to-sample thermal exchange radius $r_{\mathrm{ex}}$ which is assimilated here to the circular constriction radius. ${ }^{30}$ The experimental procedure consists of applying the $3 \omega$ method scanning the SThM probe on an abrupt step made of a $200 \mathrm{~nm}$ thick oxide layer on a Si substrate. When the probe placed upon the oxide layer is shifted toward the step, the tip-to-sample thermal exchange surface is truncated, which causes an increase of the tip temperature variations until a maximum value when the contact point is at the edge of the step. Measuring the distance from the position for which the $\mathrm{V}_{3 \omega}$ signal starts to increase to the position for which the signal is maximal leads to the tip-to-sample thermal exchange radius $r_{\text {ex }}$. Here, its value has been evaluated to $r_{e x}=230 \mathrm{~nm}$. This value has double importance: on the one hand, the thermal lateral resolution depends on it and, on the other hand, it constitutes an essential value in our method to determine the NW thermal conductivity as presented in Sec. III.

\section{STHM MEASUREMENTS}

\section{A. NWs equivalent thermal resistance evaluation}

The $3 \mu \mathrm{m} \times 3 \mu \mathrm{m}$ topographical and $\left(\mathrm{V}_{3 \omega}\right)_{\text {Tip }}$ images obtained simultaneously using the $3 \omega$-SThM technique described above at a $927 \mathrm{~Hz}$ fundamental frequency $(\omega=5825 \mathrm{rad} / \mathrm{s})$ are presented in Figure 2 . The NWs jutting out above the matrix (bright parts in Figure 2(a)) offer a lower $3 \omega$ voltage (dark parts in Figure 2(b)) than the alumina matrix, consequently a lower $2 \omega$ temperature variation. This is explained by an increase of the heat flux passing from the tip to the sample in this region. The equivalent thermal resistance is then lower on the NWs than on the alumina.
From the $\left(\mathrm{V}_{3 \omega}\right)_{\text {Tip }}$ image (Figure 2(b)), we have plotted in Figure 3 the distribution of the equivalent thermal resistances measured on the alumina part of the sample and on the $\mathrm{Bi}_{2} \mathrm{Te}_{3}$ NWs. We clearly distinguish two separate distributions. The mean equivalent thermal resistance value is $\left\langle\left(\mathrm{R}_{\mathrm{eq}}\right)_{\mathrm{NW}}\right\rangle=(1.53 \pm 0.05) \times 10^{6} \mathrm{~W} / \mathrm{K}$ on the $\mathrm{NWs}$ and $\left\langle\left(\mathrm{R}_{\text {eq }}\right)_{\text {Alu }}\right\rangle=(1.75 \pm 0.02) \times 10^{6} \mathrm{~W} / \mathrm{K}$ on the alumina. The mean equivalent thermal resistance is higher on the alumina part than on the NWs, which indicates that thermal conductivity of the alumina is lower than the NW one.

Now, from these mean equivalent thermal resistance values, we want to evaluate the mean thermal conductivity of the NWs. Let us consider a NW on top of which the tip, whose SEM image is presented in the top left part of the image, is positioned to do a $3 \omega-\mathrm{SThM}$ measurement (Figure 4). The NW equivalent thermal resistance $\left(\mathrm{R}_{\mathrm{eq}}\right)_{\mathrm{NW}}$ can be modelled by 4 thermal resistances in series at the most: the tipto-sample contact thermal resistance $\mathrm{R}_{\mathrm{C}}$, the constriction resistance $R_{\text {Tip-NW }}$ of the heat flux between the tip and the $\mathrm{NW}$, the sample intrinsic thermal resistance $\mathrm{R}_{\mathrm{NW}}$, and the constriction resistance $\mathrm{R}_{\mathrm{NW} \text {-Sub }}$ of the heat flux between the NW and the substrate on which the NW is deposited

$$
\left(R_{e q}\right)_{N W}=R_{T i p-N W}+R_{c}+R_{N W}+R_{N W-S u b} .
$$

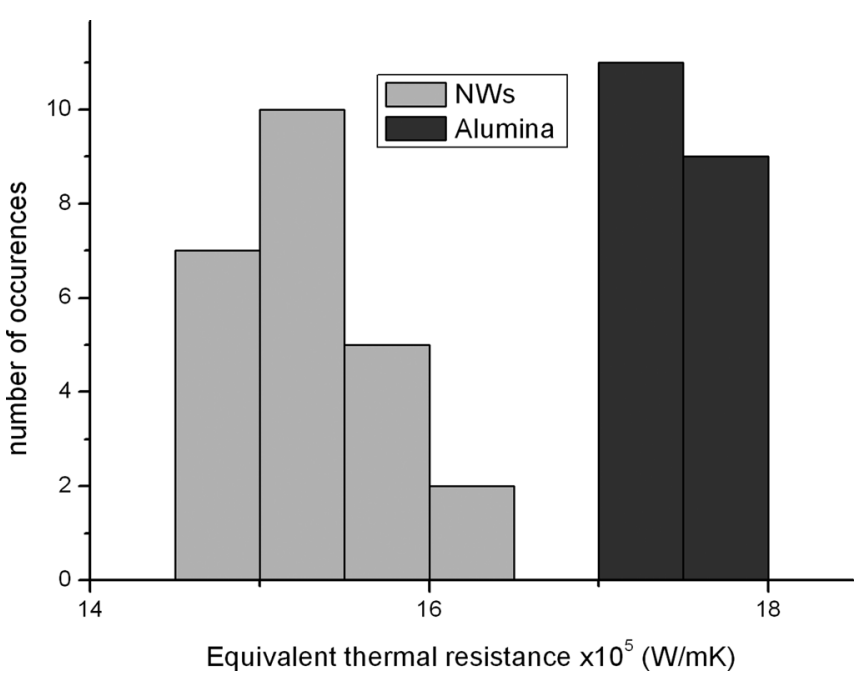

FIG. 3. Equivalent thermal resistance distribution on the alumina and NWs parts of the sample. 


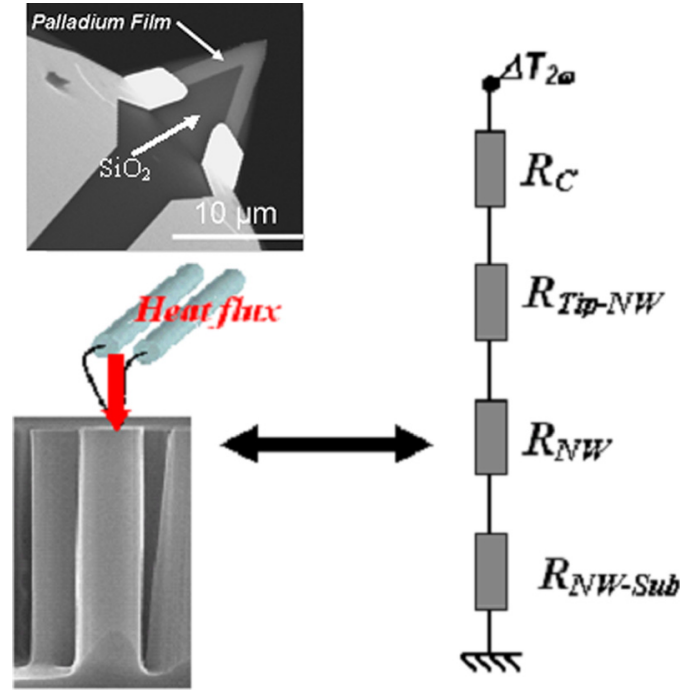

FIG. 4. Tip image (top left) and equivalent thermal schema of the thermal flux passing from the tip to a NW.

Then, to extract the mean NW thermal conductivity which is included in the intrinsic thermal resistance $\mathrm{R}_{\mathrm{NW}}$, we must determine the values of the three thermal resistances $\mathrm{R}_{\text {Tip-NW}}, \mathrm{R}_{\mathrm{C}}$, and $\mathrm{R}_{\mathrm{NW} \text {-Sub }}$ as well as the expression of the NW intrinsic thermal resistance $R_{N W}$ as a function of its thermal conductivity $\lambda_{\mathrm{NW}}$.

The constriction resistance $\mathrm{R}_{\text {Tip-NW }}$ between the tip and the NW is negligible as the thermal exchange surface is bigger than the NWs section, the diameter $\mathrm{d}_{\mathrm{NW}}$ of the probed NWs varying from 200 to $400 \mathrm{~nm}$. The constriction resistance between the NW and the substrate can be expressed as $^{32}$

$$
R_{N W-S u b}=\frac{1}{2 \lambda_{S u b} d_{N W}}
$$

with $\lambda_{\text {Sub }}$ the substrate thermal conductivity. In our case of NWs deposited on $\mathrm{Cr} / \mathrm{Au}$, the mean value of this thermal resistance is of the order of $10^{4} \mathrm{~W} / \mathrm{K}$, depending on the NW diameter value. Therefore, it is negligible compared to the mean equivalent thermal resistance $\left\langle\left(\mathrm{R}_{\mathrm{eq}}\right)_{\mathrm{NW}}\right\rangle$ measured on the NWs.

The contact thermal resistance $R_{C}$ can be evaluated measuring the equivalent thermal resistance on the porous alumina part of the thermal image. ${ }^{19,29,33}$ From 20 values measured on the porous alumina (Figure 3), we have previously deduced the mean equivalent thermal resistance $\left\langle\left(\mathrm{R}_{\text {eq }}\right)_{\mathrm{Alu}}\right\rangle=(1.75 \pm 0.02) \times 10^{6} \mathrm{~W} / \mathrm{K}$. This resistance is the sum of the mean contact thermal resistance $\left\langle R_{C}\right\rangle$ and of the constriction resistance between the tip and the alumina which can be expressed as

$$
R_{T i p-A l u}=\frac{1}{4 \lambda_{A l u} r_{e x}},
$$

where $\lambda_{\text {Alu }}$ is the thermal conductivity of the porous alumina and its value has been previously measured to be $1.3 \mathrm{~W} / \mathrm{mK} .^{34}$ Then the mean contact resistance can be esti- mated to $\left\langle R_{C}\right\rangle=(9.1 \pm 0.2) \times 10^{5} \mathrm{~W} / \mathrm{K}$. The low value of the uncertainty, which is calculated from the standard deviation of the values measured on the twenty positions on the alumina, indicates that the contact resistance does not vary much from one point to another. From the NW equivalent thermal resistances $\left(\mathrm{R}_{\mathrm{eq}}\right)_{\mathrm{NW}}$ we have measured, we can now deduce the mean intrinsic thermal resistance value $\left\langle\mathrm{R}_{\mathrm{NW}}\right\rangle=(6.01 \pm 0.41) \times 10^{5} \mathrm{~W} / \mathrm{K}$. Next step consists in deducing an estimation of the mean NW thermal conductivity $\left\langle\lambda_{\mathrm{NW}}\right\rangle$. For that purpose, we need to know the expression of the NW intrinsic thermal resistance $\mathrm{R}_{\mathrm{NW}}$ as a function of $\lambda_{\mathrm{NW}}$.

\section{B. NW composite thermal model and thermal conductivity}

Classically, $\mathrm{R}_{\mathrm{NW}}$ is assumed to be given by the conductive thermal resistance

$$
R_{N W}=\frac{1}{\lambda_{N W}} \frac{L}{S}
$$

of a thermal conductor of length $\mathrm{L}$ and section $\mathrm{S}$. Let us recall that the NWs have the c-axis oriented along their length, i.e., perpendicular to the alumina template surface. In addition, the measurements have been made in the same direction. The measured $\lambda_{\mathrm{NW}}$ therefore corresponds to the thermal conductivity along the NWs length. According to Eq. (5), $\mathrm{R}_{\mathrm{NW}}$ should vary from one NW to another depending on the NW section, given that all the probed NWs have the same length while their diameter distribution is very large. Nevertheless, this behaviour is not observed since when dividing the section by a factor 2 , the $\mathrm{R}_{\mathrm{NW}}$ value is not quite affected. The same conclusion can be reached noting that the dispersion on the $\mathrm{R}_{\mathrm{NW}}$ value is relatively low (7\%) for a population with a $25 \%$ section dispersion.

Besides, the expression (5) would lead to a $410 \mathrm{~W} / \mathrm{mK}$ NW thermal conductivity. Therefore, this expression may not be adapted to our configuration. Then, two hypothesis can be considered. The first one is that the length $\mathrm{L}$ appearing in expression (5) is not correctly evaluated. Indeed, the NWs are relatively long $(37 \mu \mathrm{m})$ and the thermal diffusion length in this material at $927 \mathrm{~Hz}$ is only $11 \mu \mathrm{m}$, taking into account a $6.9 \times 10^{-7} \mathrm{~m}^{2} \mathrm{~s}^{-1}$ thermal diffusivity of such NWs identified by Borca-Tasciuc et al. ${ }^{35}$ Then, we can assume the heat only diffuses over the thermal diffusion length and not along the total NW length. Consequently, we should take into account the thermal diffusion length in expression (5) instead of the real length of the NW.

Nevertheless, even in this case, the NW mean thermal conductivity remains very high. In addition, since the thermal diffusion length depends on the frequency, the measured $\mathrm{R}_{\mathrm{NW}}$ should also depend on it. Thus, we have performed thermal images at $309 \mathrm{~Hz}$. Since the frequency has been divided by 3 , the thermal diffusion length has increased to reach $19 \mu \mathrm{m}$ and the mean NW thermal resistance $R_{\mathrm{NW}}$ should then vary by a factor $\sqrt{3}$ but it only changes from $\left\langle\mathrm{R}_{\mathrm{NW}}\right\rangle=(6.01 \pm 0.41) \times 10^{5} \mathrm{~W} / \mathrm{K}$ at $927 \mathrm{~Hz}$ to $\left\langle\mathrm{R}_{\mathrm{NW}}\right\rangle=(5.80 \pm 0.44) \times 10^{5} \mathrm{~W} / \mathrm{K}$ at $309 \mathrm{~Hz}$. Measurements done at two other frequencies (103 and $2781 \mathrm{~Hz}$ ) have 
confirmed that this hypothesis does not seem to work as expected.

In the second hypothesis, we do not consider the NWs as individual elements but as part of a composite made of $\mathrm{Bi}_{2} \mathrm{Te}_{3} \mathrm{NWs}$ and the alumina matrix. This can be justified by the fact that the NWs are in partial contact with the alumina matrix as it can be seen in the SEM images (Figure 1) and not isolated from it. Moreover, the thermal conductivities of the alumina and of the NWs are expected to be of the same order. Therefore, when the tip is in contact with a NW, part of the heat flux propagating along a NW passes from the NW to the matrix. In addition, the thermal exchange surface is bigger than the NWs section. Then, the tip heats not only the NW but also the surrounding alumina matrix at the same time. In this case, the thermal resistance measured when scanning a NW is not given by expression (5) but by the expression of a constriction resistance on a semi-infinite effective medium

$$
R_{N W}=\frac{1}{4 \lambda_{c} r_{e x}}
$$

where $\lambda_{\mathrm{C}}$ is the composite thermal conductivity. From the various measurements done on the images obtained at different frequencies, we can now deduce $\left\langle\lambda_{\mathrm{C}}\right\rangle=(1.68 \pm 0.20)$ $\mathrm{W} / \mathrm{mK}$. This value has been obtained using the measurements made on $49 \mathrm{NWs}$ whose thermal conductivity distribution is presented in Figure 5. We can note a low uncertainty corresponding to a low dispersion on the measurements. As expected from Figure 3, this thermal conductivity is higher than the one of the porous alumina. This value, which is of great importance since it constitutes the thermal conductivity of the functional device, could be reduced if using another matrix with a lower thermal conductivity: Biswas et al. ${ }^{16}$ have shown that using epoxy resin SU8 instead of porous anodic alumina, the composite thermal conductivity of $200 \mathrm{~nm}$ diameter $\mathrm{Bi}_{2} \mathrm{Te}_{3} \mathrm{NW} /$ matrix could be reduced from $1.4 \mathrm{~W} / \mathrm{mK}$ with the alumina matrix to $1.1 \mathrm{~W} / \mathrm{mK}$ with the SU-8 matrix.

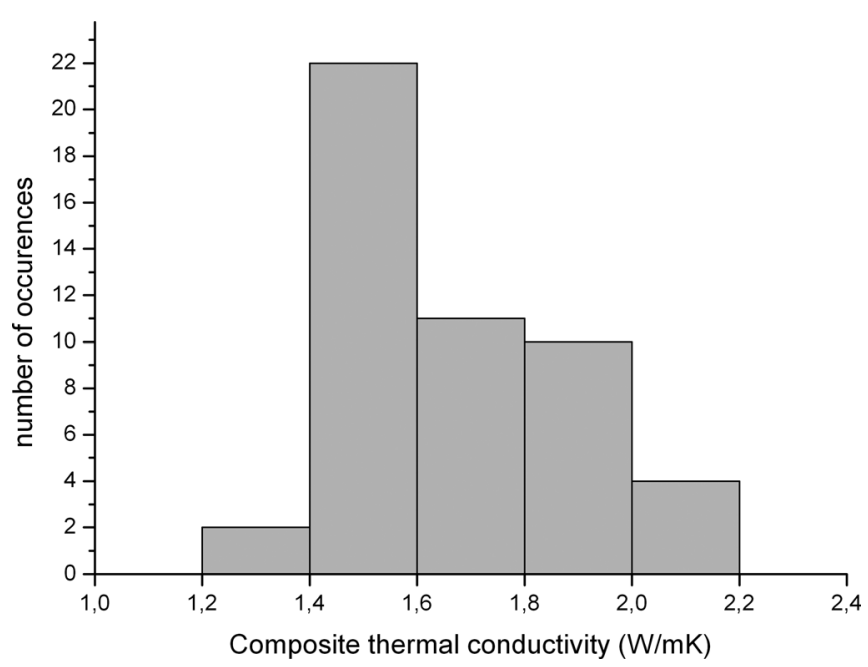

FIG. 5. Composite thermal conductivity distribution on around 50 NWs.

\section{NANOWIRES THERMAL CONDUCTIVITY AND DISCUSSION}

From the composite thermal conductivity, we can now assess the NW thermal conductivity using the effective medium theory, ${ }^{22}$ neglecting the thermal resistance at the alumina/NW interface since the NWs are in contact with the alumina and both media are expected to have close thermal conductivity values. Then, the thermal conductivity of the composite made of the NW array and the matrix can be estimated as ${ }^{16,22}$

$$
\lambda_{c}=x \lambda_{N W}+(1-x) \lambda_{m}
$$

where $\mathrm{x}$ is the areal packing density of the NW array, $\lambda_{\mathrm{NW}}$ and $\lambda_{\mathrm{m}}$ are, respectively, the thermal conductivities of the NWs and matrix. From the SEM images, we have evaluated the percentage of NWs to be $41 \%$ and the percentage of alumina to be $59 \%$. With the thermal conductivity of the solid alumina matrix evaluated to $\lambda_{\mathrm{m}}=1.9 \mathrm{~W} / \mathrm{mK}$ at room temperature, ${ }^{34}$ we can deduce from Eq. (7) that $\lambda_{\mathrm{NW}}=(1.37 \pm 0.20)$ $\mathrm{W} / \mathrm{mK}$.

In literature, other techniques developed to measure single NWs or whole arrays of NWs can be found. In single NWs thermal conductivity experiments, the NW is generally suspended onto the electrodes of a thermal microchip. ${ }^{13,14,36}$ These experiments have reported data for $\mathrm{Bi}_{2} \mathrm{Te}_{3}$ at room temperature of $1.3 \mathrm{~W} / \mathrm{mK}$ for $55 \mathrm{~nm}$ diameter NWs. ${ }^{36}$ It is worth mentioning here that for this kind of experiments, the matrix has to be dissolved and this process, having the NWs in air, leads to the oxidation of the surface of the NWs. ${ }^{37}$ Other techniques, ${ }^{15,16,22}$ based on the effective medium theory, allow the measurement of the thermal conductivity of an array of NWs inside a matrix, obtaining the thermal conductivity of the whole structure. Then, the NW thermal conductivity can be estimated. Reported data for $\mathrm{Bi}_{2} \mathrm{Te}_{3} \mathrm{NWs}$ measured with this technique are $0.75 \mathrm{~W} / \mathrm{mK}$ for $120 \mathrm{~nm}$ diameter $^{15}$ and $1.44 \mathrm{~W} /$ $\mathrm{mK}$ for $200 \mathrm{~nm}$ diameter. ${ }^{16}$

The advantage of the $3 \omega-\mathrm{SThM}$, in comparison to these techniques, is the possibility to measure the thermal conductivity of single NWs embedded in the matrix whilst at the same time the thermal conductivity of the composite is locally measured. Moreover, a topographic picture of the surface of the sample can be also obtained to assure the local measurement.

As for the thermal conductivity value we have obtained, it lies within the range of the reported data mentioned above. Yet, we cannot rigorously compare these results because of the differences within the techniques and the samples. In particular, measurements with a thermal microchip and with $3 \omega$-SThM must be compared with precaution for two main reasons: first, the reported samples have diameters far smaller than ours and second, the thermal microchip technique probes one single isolated $\mathrm{NW}$, whereas $3 \omega-\mathrm{SThM}$ probes an assembly of NWs embedded in a matrix. Nevertheless, comparing our value measured for NWs by the $3 \omega$-SThM with identical NWs measured with a thermal microchip could enable to study the influence of the surrounding matrix or the oxidation of the single NW outside the matrix. Comparing $3 \omega-\mathrm{SThM}$ results with the values 
measured by the techniques based on the effective medium theory seems more appropriate since in both cases, the samples are embedded in a matrix. Even if no identical sample (same NW length, NW diameter, matrix and growing process) has been reported in literature, our value is in particular good agreement with the value given in Ref. 16, which corresponds to NWs with diameter and length of the same order than ours embedded in an alumina matrix.

Whatever the method, in all cases, these measurements show a small thermal conductivity reduction in comparison with the bulk $\mathrm{Bi}_{2} \mathrm{Te}_{3}$ value, as expected from nanostructuration. Molecular dynamics simulations ${ }^{38}$ have also predicted a thermal conductivity reduction limited to $20 \%$ (in comparison with the bulk thermal conductivity), even for $30 \mathrm{~nm}$ diameter $\mathrm{Bi}_{2} \mathrm{Te}_{3}$ NWs. This thermal conductivity reduction is less pronounced than in Si for which the thermal conductivity of $56 \mathrm{~nm}$ diameter NWs has been measured to be around $25 \mathrm{~W} / \mathrm{mK}$ (Ref. 13) at room temperature, that is, 6 times lower than the bulk thermal conductivity. In this case, the phonon mean free path $(250 \mathrm{~nm}$ in bulk $\mathrm{Si}$ at $300 \mathrm{~K})$ is higher than the NW diameter, favouring phonon boundary scattering and a thermal conductivity reduction. In bulk bismuth telluride, the phonon mean free path is only $3 \mathrm{~nm},{ }^{36}$ which is much smaller than every diameter of the studied NWs. Therefore, the impact of phonon boundary scattering is in our case much smaller than in Si NWs, leading to a limited thermal conductivity reduction.

\section{CONCLUSIONS}

We have evaluated the thermal conductivity of a $\mathrm{Bi}_{2} \mathrm{Te}_{3}$ NWs array using SThM in a $3 \omega$ experimental configuration. The classical thermal model of the NWs based on a conductive intrinsic thermal resistance of the NWs could not be applied in our case, leading us to use an effective medium model. We could then deduce the mean thermal conductivity of the composite made of NWs embedded in alumina with a good precision: $\left\langle\lambda_{\mathrm{C}}\right\rangle=(1.68 \pm 0.20) \mathrm{W} / \mathrm{mK}$. The NWs thermal conductivity was then estimated to $\left\langle\lambda_{\mathrm{NW}}\right\rangle$ $=(1.37 \pm 0.20) \mathrm{W} / \mathrm{mK}$.

Up to now, it was possible to measure either the thermal conductivity of single NWs outside their supporting matrix, e.g., using a thermal microchip, or the thermal conductivity of NWs embedded in a matrix as a whole, to later extract the NWs thermal conductivity. ${ }^{15,16,36}$ However, in this work, we present for the first time the measurement of the thermal conductivity of single $\mathrm{Bi}_{2} \mathrm{Te}_{3}$ NWs embedded in a matrix by $3 \omega$-SThM technique, which allows the extraction of information not only of the NWs but also about the local thermal conductivity of the composite. Moreover, it must be noticed that a topographic image of the NW is also obtained, granting the local measurement of the technique. The reason why we propose this technique as an alternative to those reported for thermal conductivity measurements is not only its local resolution and analysis but also its nondestructive nature and the versatility of the samples that can be measured.

Next step could consist in using pump-probe measurements $^{39}$ on the same sample in order to estimate the thermal conductivity of $\mathrm{Bi}_{2} \mathrm{Te}_{3} \mathrm{NWs}$ and compare the results with the $3 \omega-\mathrm{SThM}$ values. New samples with another kind of matrix with lower thermal conductivity, such as epoxy resin, could also be investigated in order to reduce the whole composite thermal conductivity. Finally, another interesting approach could be to create a sample with $\mathrm{Bi}_{2} \mathrm{Te}_{3} \mathrm{NWs}$ isolated from the matrix to directly access the NW thermal conductivity by $3 \omega$-SThM and/or pump-probe measurements.

\section{ACKNOWLEDGMENTS}

The authors acknowledge Bernard Plano from the IMS (Bordeaux 1) and Jaime Martin Pérez from the IMM-CSIC (Madrid) for the SEM images.

This work has been supported by the ANR PNANO and by ERC Starting Grant Nano-TEC number 240497. M.M and O.C. wants to acknowledge CSIC and European Social Fund for financial support by JAE-Pre and JAE-Doc.

${ }^{1}$ W. Wang, G. Zhang, and X. Li, J. Phys. Chem. C 112, 15190 (2008).

${ }^{2}$ N. Shirato, J. Strader, A. Kumar, A. Vincent, P. Zhang, A. Karakoti, P. Nacchimuthu, H.-J. Cho, S. Seal, and R. Kalyanaraman, Nanoscale 3, 1090 (2011)

${ }^{3}$ X. Feng, X. Huang, and X. Wang, Nanotechnology 23, 185701 (2012).

${ }^{4}$ P. Kim, L. Shi, A. Majumdar, and P. L. McEuen, Phys. Rev. Lett. 87, 215502 (2001).

${ }^{5}$ L. Shi, J. Zhou, P. Kim, A. Bachtold, A. Majumdar, and P. L. McEuen, J. Appl. Phys. 105, 104306 (2009).

${ }^{6}$ S. Zhao, Q. Wang, Z. Mi, S. Fathololoumi, T. Gonzalez, and M. P. Andrews, Nanotechnology 23, 415706 (2012).

${ }^{7}$ S. Volz and G. Chen, Appl. Phys. Lett. 75, 2056 (1999).

${ }^{8}$ Z. Wang and N. Mingo, Appl. Phys. Lett. 97, 101903 (2010).

${ }^{9}$ X. Chen, Y. Wang, Y. Ma, T. Cui, and G. Zou, J. Phys. Chem. C 113, 14001 (2009).

${ }^{10}$ A. J. H. McGaughey, E. S. Landry, D. P. Sellan, and C. H. Amon, Appl. Phys. Lett. 99, 131904 (2011).

${ }^{11}$ N. Mingo, Phys. Rev. B 68, 113308 (2003).

${ }^{12}$ D. Lacroix, K. Joulain, D. Terris, and D. Lemonnier, Appl. Phys. Lett. 89, 103104 (2006).

${ }^{13}$ D. Li, Y. Wu, P. Kim, L. Shi, P. Yang, and A. Majumdar, Appl. Phys. Lett. 83, 2934 (2003).

${ }^{14}$ A. I. Hochbaum, R. Chen, R. Diaz Delgado, W. Liang, E. C. Garnett, M. Najarian, A. Majumdar, and P. Yang, Nature 451, 163 (2008).

${ }^{15}$ C.-L. Chen, Y.-Y. Chen, S.-J. Lin, J. C. Ho, P.-C. Lee, C.-D. Chen, and S. R. Harutyunyan, J. Phys. Chem. C 114, 3385 (2010).

${ }^{16}$ K. G. Biswas, T. D. Sands, B. A. Cola, and X. Xu, Appl. Phys. Lett. 94, 223116 (2009).

${ }^{17}$ T. Souier, G. Li, S. Santos, M. Stefancich, and M. Chiesa, Nanoscale 4, 600 (2012).

${ }^{18}$ L. Shi, O. Kwon, A. C. Miner, and A. Majumdar, J. Microelectromech. Syst. 10, 370 (2001)

${ }^{19}$ M. Hinz, O. Marti, B. Gotsmann, M. A. Lantz, and U. Dürig, Appl. Phys. Lett. 92, 043122 (2008).

${ }^{20}$ C. D. S. Brites, P. P. Lima, N. J. O. Silva, A. Milla, V. S. Amaral, F. Palacio, and L. D. Carlos, Nanoscale 4, 4799 (2012).

${ }^{21}$ J. H. Rice, Nanoscale 2, 660 (2010).

${ }^{22}$ A. I. Persson, Y. K. Koh, D. G. Cahill, L. Samuelson, and H. Linke, Nano Lett. 9, 4484 (2009).

${ }^{23}$ P. Magri, C. Boulanger, and J. M. Lecuire, J Mater. Chem. 6, 773 (1996).

${ }^{24}$ M. S. Martin-Gonzalez, A. L. Prieto, R. Gronsky, T. Sands, and A. M. Stacy, J. Electrochem. Soc. 149, C546-C554 (2002).

${ }^{25}$ A. L. Prieto, M. S. Sander, M. S. Martin-Gonzalez, R. Gronsky, T. Sands, and A. M. Stacy, J. Am. Chem. Soc. 123, 7160 (2001).

${ }^{26}$ D. G. Cahill, Rev. Sci. Instrum. 61, 802 (1990).

${ }^{27}$ L. Shi, S. Plyasunov, A. Bachtold, P. L. McEuen, and A. Majumdar, Appl. Phys. Lett. 77, 4295 (2000).

${ }^{28}$ S. Lefèvre and S. Volz, Rev. Sci. Instrum. 76, 033701 (2005).

${ }^{29}$ E. Puyoo, S. Grauby, J.-M. Rampnoux, E. Rouvière, and S. Dilhaire, J. Appl. Phys. 109, 024302 (2011). 
${ }^{30}$ E. Puyoo, S. Grauby, J.-M. Rampnoux, E. Rouvière, and S. Dilhaire, Rev. Sci. Instrum. 81, 073701 (2010).

${ }^{31}$ S. Lefèvre, S. Volz, J.-B. Saulnier, C. Fuentes, and N. Trannoy, Rev. Sci. Instrum. 74, 2418 (2003).

${ }^{32}$ R. S. Prasher, Nano Lett. 5, 2155 (2005).

${ }^{33}$ Y. Zhang, C. L. Hapenciuc, E. E. Castillo, T. Borca-Tasciuc, R. J. Mehta, C. Karthik, and G. Ramanath, Appl. Phys. Lett. 96, 062107 (2010).

${ }^{34}$ D.-A. Borca-Tasciuc and G. Chen, J. Appl. Phys. 97, 084303 (2005).
${ }^{35}$ D.-A. Borca-Tasciuc, G. Chen, A. Prieto, M. S. Martin-Gonzalez, A. Stacy, T. Sands, M. A. Ryan, and J. P. Fleurial, Appl. Phys. Lett. 85, 6001 (2004).

${ }^{36}$ A. Mavrokefalos, A. L. Moore, M. T. Pettes, L. Shi, W. Wang, and X. Li, J. Appl. Phys. 105, 104318 (2009).

${ }^{37}$ E. J. Menke, M. A. Brown, Q. Li, J. C. Hemminger, and R. M. Penner, Langmuir 22, 10564 (2006).

${ }^{38}$ B. Qiu, L. Sun, and X. Ruan, Phys. Rev. B 83, 035312 (2011).

${ }^{39}$ S. Dilhaire, G. Pernot, G. Calbris, J.-M. Rampnoux, and S. Grauby, J. Appl. Phys. 110, 114314 (2011). 